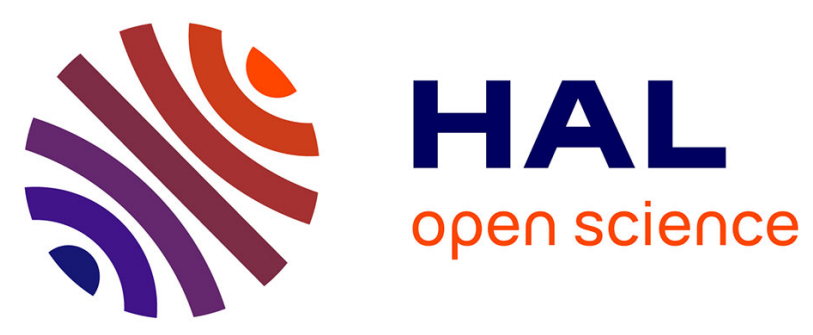

\title{
Use of partially hydrolysed formula in infancy and incidence of eczema, respiratory symptoms or food allergies in toddlers from the ELFE cohort
}

Camille Davisse-Paturet, Chantal Raherison, Karine Adel-Patient, Amandine Divaret-Chauveau, Corinne Bois, Marie-Noëlle Dufourg, Sandrine Lioret, Marie-Aline Charles, Blandine de Lauzon-Guillain

\section{To cite this version:}

Camille Davisse-Paturet, Chantal Raherison, Karine Adel-Patient, Amandine Divaret-Chauveau, Corinne Bois, et al.. Use of partially hydrolysed formula in infancy and incidence of eczema, respiratory symptoms or food allergies in toddlers from the ELFE cohort. Pediatric Allergy and Immunology, 2019, 10.1111/pai.13094 . inserm-02305833

\section{HAL Id: inserm-02305833 https://www.hal.inserm.fr/inserm-02305833}

Submitted on 4 Oct 2019

HAL is a multi-disciplinary open access archive for the deposit and dissemination of scientific research documents, whether they are published or not. The documents may come from teaching and research institutions in France or abroad, or from public or private research centers.
L'archive ouverte pluridisciplinaire HAL, est destinée au dépôt et à la diffusion de documents scientifiques de niveau recherche, publiés ou non, émanant des établissements d'enseignement et de recherche français ou étrangers, des laboratoires publics ou privés. 


\section{TITLE'S PAGE}

\section{Full title:}

Use of partially hydrolysed formula in infancy and incidence of eczema, respiratory symptoms or food allergies in toddlers from the ELFE cohort

\section{Authors:}

Camille Davisse-Paturet ${ }^{1,2}$, Chantal Raherison ${ }^{3}$, Karine Adel-Patient ${ }^{4}$, Amandine Divaret-

Chauveau $^{5,6}$, Corinne Bois ${ }^{7,8}$, Marie-Noëlle Dufourg ${ }^{7}$, Sandrine Lioret ${ }^{1,2}$, Marie-Aline

Charles $^{1,2,7 *}$, Blandine de Lauzon-Guillain ${ }^{2,9 *}$

* The two last authors have equally contributed to this work

\section{Affiliations:}

${ }^{1}$ INSERM, UMR1153 Centre for Research in Epidemiology and StatisticS (CRESS),

Research team on EARly life Origins of Health (EAROH), Paris, France

${ }^{2}$ Université de Paris, Paris, France

${ }^{3}$ Bordeaux University, Inserm, Bordeaux Population Health Research Center, Team

EPICENE, UMR 1219, Bordeaux, France

${ }^{4}$ UMR Service de Pharmacologie et Immunoanalyse, CEA, INRA, Université Paris-Saclay, Gif-sur-Yvette, France

${ }^{5}$ Unité d'allergologie pédiatrique, Hôpital d'enfants, CHRU de Nancy, France

${ }^{6}$ EA3450, Université de Lorraine, Vandoeuvre les Nancy, France

${ }^{7}$ Unité mixte Inserm-Ined-EFS Elfe, INED, Paris, France

${ }^{8}$ Service départemental de PMI, Conseil départemental des Hauts-de-Seine, Nanterre, France

${ }^{9}$ INRA, U1125 Centre for Research in Epidemiology and StatisticS (CRESS), Research team on EARly life Origins of Health (EAROH), Paris, France 


\section{Running title}

Infant formula and allergy-related symptoms

\section{Corresponding author:}

Blandine de Lauzon-Guillain

INSERM CRESS Eq 6 EAROH,

Hôpital Paul Brousse, Bâtiment 15/16

16 avenue Paul Vaillant Couturier

94807 Villejuif Cedex

France

$+33145595019$

blandine.delauzon@inserm.fr

\section{Word count}

2916words from the start of the introduction to the end of conclusion

Figures: 3

Tables: 2

Appendices: 2 supplementary methods, 5 tables, 1 supplementary material 


\section{MAIN TEXT FILE}

\section{Potential conflict of interest statement}

CR reports grants from Bordeaux University Foundation during the conduct of the study. Other authors declare no support from any organisation for the submitted work.

ADC reports other from Mead Jonhson, other from Nutricia, other from Novalac, and other from Sodilac outside the submitted work; CR reports personal fees from Astra Zeneca, personal fees from Chiesi, personal fees from ALK, personal fees from Boehringer Ingelheim, personal fees from Glaxo SmithKline, personal fees from MundiPharma, and personal fees from Novartis outside the submitted work; BLG reports grants from French national research agency (ANR), and personal fees from French Agency for Food, Environmental and Occupational Health \& Safety (ANSES) outside the submitted work; other authors declare no financial relationships with any organisations that might have an interest in the submitted work in the previous three years. Authors declare no other relationships or activities that could appear to have influenced the submitted work.

\section{Financial support}

The ELFE survey is a joint project between INED (Institut National d'Etudes Démographiques), INSERM (Institut National de la Santé et de la Recherche Médicale), EFS (Etablissement Français du Sang), InVS (Institut de Veille Sanitaire), INSEE (Institut National de la Statistique et des Etudes Economiques), the Ministry of Health (DGS, Direction Générale de la Santé), the Ministry of Environment (DGPR, Direction Générale de la Prévention des Risques), the Ministry of Health and Employment (DREES, Direction de la Recherche, des Etudes, de l'Evaluation et des Statistiques), and the CNAF (Caisse Nationale des Allocations Familiales), with the support of the Ministry of Research and CCDSHS (Comité de Concertation pour les Données en Sciences Humaines et Sociales) and the 
Ministry of Culture (DEPS, Département des études, de la prospective et des statistiques). As part of the RECONAI platform, the study received state funding from the ANR within the framework of the "Future Investments" program (reference: ANR-11- EQPX-0038). The funders had no role in the study design, data collection and analysis, decision to publish, or preparation of the manuscript.

\section{Abbreviations}

CMPA: cow's milk protein allergy; ELFE: étude longitudinale française depuis l'enfance; FA: food allergy; eHF: extensively hydrolysed formula; nHF: non-hydrolysed formula; pHFnon-HA: partially hydrolysed formula without hypoallergenic label; pHF-HA: partially hydrolysed formula with hypoallergenic label; WHO: World Health Organization.

\section{Authors' contributions}

The corresponding author attests that all listed authors meet authorship criteria and that no others meeting the criteria have been omitted.

Ms Davisse-Paturet conceptualized and designed the study, conducted the statistical analyses, interpreted the results and drafted the initial manuscript. Dr Raherison contributed to the study design and critically reviewed the manuscript. Drs Adel-Patient, Divaret-Chauveau and Lioret contributed to the interpretation of the results and critically reviewed the manuscript. Drs Bois and Dufourg designed the data collection instruments, supervised data collection and data management and critically reviewed the manuscript. Drs Charles and de LauzonGuillain conceptualized and designed the study, contributed to the interpretation of the results, reviewed and revised the manuscript.

All authors approved the final manuscript as submitted and agree to be accountable for all aspects of the work. 


\section{What is already known on this topic}

Partially hydrolyzed infant formulas are designed to prevent allergy development in infants with a family history of allergy. However, their actual efficacy in allergy prevention remains controversial.

\section{What this study adds}

In this study, the use of partially hydrolysed formula was not related to a lower risk of eczema, food allergies or respiratory symptoms compared to regular formula. Even, our results suggest that partially hydrolyzed formulas might be related to a higher risk of food allergy and respiratory symptoms development up to 2 years or age. 


\section{$\underline{\text { Abstract }}$}

Background and objectives. Partially hydrolysed formulas (pHF) are recommended in nonbreastfed infants with familial history of allergy to prevent allergy development. However, recent meta-analysis does not provide strong support for their protective effect. The present work assesses the links between 2-month infant formula use and the incidence of eczema, respiratory symptoms or food allergies (FA) up to 2 years of age.

Methods. The nationwide ELFE birth cohort is a population-based study from mainland France. Infant feeding (breast milk only, partially-hydrolysed formula with (pHF-HA) or without a hypoallergenic label (pHF-non-HA) and non-hydrolysed formula (nHF)) was reported at 2 months. Eczema, FA and respiratory symptoms such as wheezing and asthma were reported at 2 months, 1 year and 2 years. Infant with prior FA at 2 months were excluded from analyses.

Results. Among 11,720 infants, those who received only breast milk at 2 months were at lower risk of eczema at 1 year than those who received $\mathrm{nHF}(\mathrm{OR}[95 \% \mathrm{CI}]=0.78[0.65-0.94]$ in non-at-risk infants; $0.86[0.75-0.98]$ in at-risk infants). The use of pHF-HA, compared with $\mathrm{nHF}$, at 2 months was related to higher risk of wheezing at 1 year in at-risk infants (1.68[1.24$2.28])$, and higher risk of FA at 2 years both in non-at-risk infants $(3.78[1.52-9.41])$ and in atrisk infants $(2.31[1.36-3.94])$.

Conclusions. In this nationwide study, pHF-HA use was not associated with a lower risk of any of the studied outcomes. Quite the reverse, it was associated with a higher risk of wheezing and FA. This should be confirmed in further studies.

\section{Keywords}

Infancy, breast feeding, formula feeding, partially hydrolysed formula, eczema, wheezing, asthma, food allergy 


\section{MAIN TEXT}

\section{Introduction}

Allergic diseases have become a public health problem. Several studies have reported increases over time in the prevalence of allergic diseases ${ }^{1-5}$, with many factors, ranging from genetic to environmental characteristics, linked to allergic events ${ }^{6}$. Early nutrition is one of the modifiable factors frequently mentioned in association with food allergies (FA) and other allergic diseases ${ }^{7-10}$.

Current guidelines on infant feeding recommend exclusive breast feeding for the first 6 months of life, or at least 4 months, ${ }^{10,11}$ and introduction of complementary foods not before 4 months but not delayed after 6 months, even for allergenic foods ${ }^{12}$. Although the overall benefits of breast feeding on children's health and development are clearly established, its specific benefit for allergy prevention remains controversial ${ }^{13-15}$.

Furthermore, the use of infant formula with documented preventive effect against allergy development is recommended for the first 4 months among non-exclusively breast fed infants with a familial history of allergy (i.e., with at least one parent or sibling with an allergy history $)^{10}$.

Regarding formula based on partially hydrolysed protein $(\mathrm{pHF})$, even if some systematic reviews highlighted a modest protective effect ${ }^{16,17}$, other meta-analyses do not provide strong support for the existence of benefit on allergy prevention ${ }^{18,19}$ and the recent Cochrane Review found no evidence to support the use of these pHF compared to cow's milk protein formula to prevent allergic diseases among non-exclusively breast fed infants ${ }^{20}$.

Recently, the Swiss Society of Paediatrics withdrawn the guideline of pHF use among nonexclusively breast fed infants with familial history of allergy ${ }^{21}$ and an updated clinical report from the American Academy of Pediatrics concluded to a lack of evidence regarding the 
protective role of $\mathrm{pHF}$ or extensively hydrolysed formulas (eHF) in the prevention of atopic diseases $^{22}$.

Moreover, recent data suggest that pHF are more widely used than recommended in current guidelines $^{23}$. Some of these pHF have an "hypoallergenic" label without documented preventive effect against allergy development, as a clinical trial supporting the protective effect of a specific infant formula with HA label will be mandatory only from $2020^{24}$. Thus, it is difficult for parents to distinguish infant formula with documented preventive effect across the large range of available infant formulas with HA label. Finally, only a minority of infants receiving such a formula had familial history of allergy ${ }^{23}$.

In this context, the aim of this study was to examine the associations between the use of $\mathrm{pHF}$ in infancy, in real-life conditions of use, and the incidence of eczema, respiratory symptoms or FA in toddlerhood, with a particular focus on infant formula with a HA label.

\section{$\underline{\text { Subjects and methods }}$}

\section{Study design}

This analysis is based on data from the ELFE (Etude Longitudinale Française depuis l'Enfance) study, a multidisciplinary nationwide birth cohort including 18,329 children born in 2011 in 320 participating maternity units among a random sample of 349 in mainland France $^{25}$. Inclusion began in April 2011 and took place during 25 selected recruitment days over four waves of four to eight days each that covered all four seasons. Inclusion criteria were as follows: singleton or twins born after 33 weeks of gestation, to mothers aged 18 years or older and not planning to move outside of metropolitan France in the next 3 years.

Participating mothers had to provide written consent for their own and their child's participation. Fathers signed the consent form for the child's participation when present at inclusion or were informed about their rights to oppose it. The ELFE study was approved by 
the Advisory Committee for Treatment of Health Research Information (Comité Consultatif sur le Traitement des Informations pour la Recherche en Santé), the National Data Protection Authority (Commission Nationale Informatique et Libertés), and the National Statistics Council.

\section{Data collection}

\section{Infant milk feeding}

Parents were interviewed by telephone at 2 months post-partum on various subjects including infant milk feeding (only breast milk, only infant formula, combination of breast and formula milk). Parents using formula for feeding were asked to report its brand and name. Formulas were then classified as previously described ${ }^{23}$. The different groups of infant formula reported in the ELFE study are formula based on non-hydrolysed proteins (nHF), partially hydrolysed proteins, extensively hydrolysed proteins, and free amino acids (AAF); the latter two types being specifically designed for treatment of cow's milk protein allergy (CMPA) ${ }^{26}$. By definition, infant formula with a HA label were based on partially hydrolysed proteins. However, in France, some infant formulas without the HA label (pHF-non-HA) also contained partially hydrolysed proteins, probably with a lower level of hydrolysis. As the HA label suggests a protective effect on allergy development, we decided to consider separately pHF according to the claim of a HA label.

Infants receiving both breast milk and formula were classified according to the type of formula they received. Exclusively breast fed infant were considered as a separate group.

\section{Allergic events}

During the 2-month telephone interview, parents reported any medical diagnosis of cow's milk protein allergy (CMPA) and if the infant had had eczema or wheezing since birth. During the 1-year interview, they reported medical diagnosis of eczema and wheezing from the last follow-up. During the 2-year interview, they reported medical diagnosis of eczema, 
wheezing, and asthma attack in the last 12 months, as well as medical advice to avoid certain foods due to FA. At the 2-year follow-up, a questionnaire was also sent to the child's primary care physician, including diagnosis of FA as well as the method used to confirm food sensitization (IgE or skin test).

\section{Other variables}

Data collection of familial characteristics has been previously presented ${ }^{23}$. The large data collection allows accounting for the main risk factors of allergy-related diseases, in particular familial history of allergy, child's sex, C-section delivery, maternal smoking during pregnancy, any breastfeeding duration, collective care attendance, siblings, city size (considered as a proxy of air pollution). Age at complementary food introduction ${ }^{27}$ was not considered in the main analyses, as it could not formally be considered as a confounding factor, but it was introduced as an additional adjusting variable in sensitivity analyses. Details on the data collection process are available in supplementary method 1.

\section{Study sample}

The sample selection was described in Figure 1. In particular, the main analyses were based on complete cases and, in order to limit reverse causation bias, all reported CMPA cases at 2 months were withdrawn from all analyses on association between infant feeding and child's health.

\section{Statistical analyses}

To provide representative descriptive statistics of births in 2011 in France, the descriptive analyses (allowing rates and prevalence estimations) were weighted to take the inclusion procedure, attrition, and biases related to non-consent into account. Weighting also included calibration on margins from the state register's statistical data and from the 2010 French 
National Perinatal Study for the following variables: age, region, marital status, migration status, education level, and primiparity ${ }^{28}$. This weighting was calculated at each follow-up. Families selected in our analyses were compared with their non-selected ELFE counterparts by Chi-2 tests.

As infant feeding guidelines differ according to familial allergy history, all analyses on association between infant feeding and allergy-related symptoms were stratified by this variable.

Bivariate associations between infant feeding and allergy-related symptoms were assessed by Chi-2 tests.

Multivariate associations between infant feeding and allergy-related symptoms were assessed by multinomial logistic regressions, with $\mathrm{nHF}$ as the reference group. The main analyses (Model 1) were adjusted for age at first attendance at collective care arrangement, first physician seen after discharge, maternal education level and age at first child, parental place of birth, family monthly income per consumption unit, size of town of residence, maternal smoking status, passive smoking, gestational age at birth, sex, mode of delivery, any breastfeeding duration, number of people per room living in the household, recruitment wave, maternity unit size and level, and mother's region of residence. Among infants with familial history of allergy, analyses were also adjusted for the allergic relative (parents or sibling). Because the pHF-non-HA group was too small to conduct stratified analyses for FA according to family history of allergy, these infants were excluded from FA analyses. Several sensitivity analyses were conducted to address the potential bias issues and are detailed in supplementary method 2.

All analyses were carried out with SAS software version 9.4 (SAS Institute, Cary, NC, USA). 


\section{$\underline{\text { Results }}$}

The families selected in our analyses were compared with their non-selected ELFE

counterparts for the complete case analysis as presented in Supplementary Table 1. Briefly, the non-selected women were younger and had a lower education level and lower family income than the women included in these analyses.

Table 1 presents the sample characteristics according to 2-month milk feeding, and Table 2 the weighted prevalence of allergy-related symptoms.

\section{Infant milk feeding and incidence of allergy-related symptoms at 1 and 2 years}

Supplementary Table 2presents the bivariate analyses of 2-month milk feeding and eczema, FA or respiratory symptoms at 1 and 2 years.

\section{Eczema}

Compared to nHF, infants without family history of allergy who received only breast milk at 2 months were at lower risk of eczema at 1 year. Compared to nHF, the use of pHF-HA was related to higher risk of eczema at 1 year among infants with familial history of allergy

(Figure 2). These associations disappeared after exclusion of infants with allergy-related symptoms reported at 2 months (model 2). After exclusion of infants using several infant formulas up to 2 years (model 3), the association between pHF-HA and eczema at 1 year among infants with familial history of allergy was less pronounced but remained significant. Compared to nHF, the use of pHF-HA was related to higher risk of eczema at 2 years among infants with familial history of allergy only after exclusion of infants using several formulas up to 2 years. 


\section{Food allergy}

Compared to nHF, the use of pHF-HA was related to higher risk of FA reported at 2 years, regardless of family allergy history. In our sensitivity analysis to address the potential indication bias, results were reinforced after exclusion of infants with allergy-related symptoms reported at 2 months, among infants with no family allergy history, but no more significant among infants with family allergy history. After exclusion of infants using several infant formulas up to 2 years, results were reinforced among infants with family allergy history, but no more significant among infants with no family allergy history (Figure 2). When physician-reported diagnosis of FA was considered, the association between the use of pHF-HA (compared to nHF) and FA was not significant but a similar trend was observed (OR $[95 \% \mathrm{CI}]=1.45[0.43-4.84], \mathrm{p}=0.55$ for physician-reported $\mathrm{FA}$ without use of $\mathrm{IgE}$ or skin test to confirm FA; OR [95\% CI] $=2.78[0.92-8.38], \mathrm{p}=0.069$ for physician-reported FA with use of IgE or skin test to confirm FA).

\section{Wheezing}

Compared to $\mathrm{nHF}$, the use of pHF-HA was related to higher risk of wheezing at 1 year among infants with family allergy history only. These findings were reinforced after exclusion of infants with allergy-related symptoms reported at 2 months or using several infant formulas up to 2 years (Figure 3). Compared to nHF, the use of pHF-HA was related to higher risk of wheezing at 2 years among infant with a family history of allergy only after exclusion of allergy-related symptoms reported at 2 months.

\section{Asthma attack in the second year of life}

In our main analysis, as well as in the sensitivity analysis conducted among infants consuming the same infant formula up to 2 months, infant feeding reported at 2 months was not related to asthma attack reported at 2 years. However, in the sensitivity analysis excluding infants with allergy-related symptoms reported at 2 months, compared to nHF, the use of 
pHF-HA was related to higher risk of asthma attack reported at 2 years among infants with family history of allergy (Figure 3).

\section{Sensitivity analyses}

Further adjustment on age at complementary feeding introduction (Figures 2 and 3, model 4) did not modify the association between the used of pHF-HA and the health outcomes considered in our analyses.

In analyses restricted on whey-based pHF, regardless of HA label, findings were consistent with those from the main analyses (Supplementary table 4), suggesting that the type of hydrolysed proteins used in infant formulas was not the main determinant of the association. In analyses considering infant feeding assessed at 4 months instead of 2 months, results were consistent with main analyses, except marginal changes (Supplementary table 4), suggesting that the timing of use of infant formula in these first months of life was not the main determinant of the association .

Finally, in analysis using multiple imputations to deal with missing data on potential confounders, results were consistent with those from the complete-case analysis

\section{(Supplementary table 4).}

\section{$\underline{\text { Discussion }}$}

In this nationwide French birth cohort, comparisons of different levels of protein hydrolysis showed that pHF-HA was not associated with a lower risk in any of the studied allergyrelated symptoms and was even associated with a higher risk of eczema and wheezing at 1 year in infants with family allergy history, and with a higher risk of FA at 2 years whatever the family history of allergy.

Even if the protective association of breastfeeding against atopic diseases development is not consistent through studies, a recent meta-analysis highlighted a protective effect of any type 
of breastfeeding on asthma in older children and of exclusive breastfeeding from 3 to 4 months on eczema up to 2 years ${ }^{14}$. This benefit may come from the immunomodulatory role of breastmilk ${ }^{29}$. Our results on allergy-related symptoms are in line with these findings, with a lower risk of eczema at 1 year in infants who received only breast milk at 2 months.

Regarding the protective effect on allergic diseases of protein hydrolysis in infant formula, the German Infant Nutritional Intervention (GINI), conducted among at-risk children ${ }^{30}$, showed a lower risk of atopic dermatitis during the first 3 years of life among the children who received a whey-based pHF in their first 4 months, compared to those receiving nHF, but did not highlight any protective effect on other allergic manifestation (allergic urticarial, FA with manifestation in the gastrointestinal tract or asthma $)^{30}$. Contrary with this trial, we did not highlight a lower risk of eczema related to the used of pHF. As our study was conducted in real conditions of use, we had to consider all types of pHF-HA together whereas the GINI trial was based only on whey-based pHF. The specific hydrolysis process and the types of hydrolysed proteins (whey or casein) may modulate immune system tolerance ${ }^{19,31}$. However, most pHF-HA used in the ELFE study were whey-based and the information regarding the types of hydrolysed proteins could not be obtained for the others. In sensitivity analyses conducted only on whey-based $\mathrm{pHF}$, results remained similar.

The absence of a protective association between the use of pHF-HA and allergy-related symptoms is in line with the recent Cochrane review ${ }^{20}$, but this is the first study showing a higher risk of wheezing and FA associated with the use of pHF-HA and these findings need to be confirmed in further studies. In particular, respiratory symptoms examined in the present study could have infectious origins. However, in France, "infant asthma" is defined as "any dyspneic episode with wheeze which has occurred at least 3 times since birth, regardless of age at onset, outbreak cause or history of atopy" ${ }^{32}$. Frequent wheezing in early childhood may then reflect higher susceptibility to respiratory allergies and asthma later in life ${ }^{33,34}$. 
The ELFE study provides prospective data from a broad, representative sample of births in 2011 in mainland France. The large sample size ensures statistical power for all the different outcomes and allows accounting for the main risk factors of allergy-related diseases. We have to acknowledge the risk of selection bias inherent to observational studies and complete case analyses. To address the issue of reverse causation and indication bias, we performed several sensitivity analyses and findings were not strongly modified. In particular, we found higher risk related to the use of pHF-HA among both at-risk and non-at-risk infants. Parental reports of eczema, FA, and respiratory symptoms do not achieve the same precision level as clinical examination, even if widely used ${ }^{35,36}$, and may lead to overestimation of allergy-related symptoms. However, our questions on respiratory symptoms were adapted for younger children from the questionnaire of the International Study of Asthma and Allergy in Childhood (ISAAC) ${ }^{37}$. Noteworthy, we conducted sensitivity analyses on physician-reported diagnoses of FA and results were consistent.

Finally, as parental-reported cases of CMPA were only collected at the 2-month interview, this time point was the only one allowing the exclusion of prior FA cases, necessary to account for the temporality criterion. To address the issue of duration of use of a specific infant formula, not available in our study, we conducted two sensitivity analyses, one excluding infant changing infant formula in the first 2 months of life and one considering 4month milk feeding instead of 2-month milk feeding. In both analyses, findings remained consistent.

\section{Conclusion}

A working group consensus concluded the paucity of data unable pHF to be recommended as second choice for infant feeding when breast feeding is not possible ${ }^{38}$. In this nationwide cohort, the use of pHF-HA at 2 months was not associated with a lower risk of any of the studied allergy-related symptoms up to 2 years and was even associated with a higher risk of 
wheezing, FA and, to a lesser extent, eczema. These findings must be confirmed in other studies worldwide but raise questions about the harmlessness of the large-scale pHF use and contribute to the debate around the accuracy of recommending $\mathrm{pHF}$ as a way of preventing allergy development in non-breastfed at-risk infant ${ }^{39,40}$.

\section{Acknowledgements:}

We would like to thank the scientific coordinators (M-A Charles, B Geay, H Léridon, C Bois, M-N Dufourg, JL Lanoé, X Thierry, C Zaros), IT and data managers, statisticians (A Rakotonirina, R Kugel, R Borges-Panhino, M Cheminat, H Juillard), administrative and family communication staff, study technicians (C Guevel, M Zoubiri, L Gravier, I, Milan, R Popa) of the ELFE coordination team as well as the families who gave their time for the study.

\section{$\underline{\text { References }}$}

1. Asher MI, Montefort S, Bjorksten B, et al. Worldwide time trends in the prevalence of symptoms of asthma, allergic rhinoconjunctivitis, and eczema in childhood: ISAAC Phases One and Three repeat multicountry cross-sectional surveys. Lancet. 2006;368(9537):733-743.

2. Panesar SS, Javad S, de Silva D, et al. The epidemiology of anaphylaxis in Europe: a systematic review. Allergy. 2013;68(11):1353-1361.

3. Turner PJ, Gowland MH, Sharma V, et al. Increase in anaphylaxis-related hospitalizations but no increase in fatalities: an analysis of United Kingdom national anaphylaxis data, 1992-2012. J Allergy Clin Immunol. 2015;135(4):956-963.e951.

4. Jackson KD, Howie LD, Akinbami LJ. Trends in allergic conditions among children: United States, 1997-2011. NCHS data brief. 2013(121):1-8.

5. Nwaru BI, Hickstein L, Panesar SS, Roberts G, Muraro A, Sheikh A. Prevalence of common food allergies in Europe: a systematic review and meta-analysis. Allergy. 2014;69(8):992-1007.

6. Lack G. Epidemiologic risks for food allergy. J Allergy Clin Immunol. 2008;121(6):1331-1336.

7. Du Toit G, Foong RX, Lack G. The role of dietary interventions in the prevention of IgE-mediated food allergy in children. Pediatr Allergy Immunol. 2017;28(3):222-229.

8. Høst A, Halken S, Muraro A, et al. Dietary prevention of allergic diseases in infants and small children. Pediatric Allergy and Immunology. 2008;19(1):1-4.

9. McGowan EC, Bloomberg GR, Gergen PJ, et al. Influence of early-life exposures on food sensitization and food allergy in an inner-city birth cohort. J Allergy Clin Immunol. 2015;135(1):171-U261. 
10. Muraro A, Halken S, Arshad SH, et al. EAACI food allergy and anaphylaxis guidelines. Primary prevention of food allergy. Allergy. 2014;69(5):590-601.

11. Agostoni C, Braegger C, Decsi T, et al. Breast-feeding: A commentary by the ESPGHAN Committee on Nutrition. J Pediatr Gastroenterol Nutr. 2009;49(1):112-125.

12. Fewtrell M, Bronsky J, Campoy C, et al. Complementary Feeding: A Position Paper by the European Society for Paediatric Gastroenterology, Hepatology, and Nutrition (ESPGHAN) Committee on Nutrition. J Pediatr Gastroenterol Nutr. 2017;64(1):119-132. 13. Liao SL, Lai SH, Yeh KW, et al. Exclusive breastfeeding is associated with reduced cow's milk sensitization in early childhood. Pediatric Allergy and Immunology. 2014;25(5):456-461.

14. Lodge CJ, Tan DJ, Lau MX, et al. Breastfeeding and asthma and allergies: a systematic review and meta-analysis. Acta Paediatr. 2015;104(467):38-53.

15. Bion V, Lockett GA, Soto-Ramirez N, et al. Evaluating the efficacy of breastfeeding guidelines on long-term outcomes for allergic disease. Allergy. 2016;71(5):661-670.

16. Vandenplas Y, Bhatia J, Shamir R, et al. Hydrolyzed formulas for allergy prevention. J Pediatr Gastroenterol Nutr. 2014;58(5):549-552.

17. Szajewska H, Horvath A. A partially hydrolyzed $100 \%$ whey formula and the risk of eczema and any allergy: an updated meta-analysis. The World Allergy Organization journal. 2017;10(1):27.

18. Boyle RJ, Ierodiakonou D, Khan T, et al. Hydrolysed formula and risk of allergic or autoimmune disease: systematic review and meta-analysis. BMJ. 2016;352:i974.

19. Cabana MD. The Role of Hydrolyzed Formula in Allergy Prevention. Ann Nutr Metab. 2017;70 Suppl 2:38-45.

20. Osborn DA, Sinn JK, Jones LJ. Infant formulas containing hydrolysed protein for prevention of allergic disease. Cochrane Database Syst Rev. 2018;10:CD003664.

21. Braegger CP. Recommandations pour l'alimentation des nourrissons 2017. Paediatrica. 2017;28(4):11-15.

22. Greer FR, Sicherer SH, Burks AW. The Effects of Early Nutritional Interventions on the Development of Atopic Disease in Infants and Children: The Role of Maternal Dietary Restriction, Breastfeeding, Hydrolyzed Formulas, and Timing of Introduction of Allergenic Complementary Foods. Pediatrics. 2019.

23. de Lauzon-Guillain B, Davisse-Paturet C, Lioret $S$, et al. Use of infant formula in the ELFE study: The association with social and health-related factors. Matern Child Nutr. 2018;14(1).

24. Commission E. Commission Delegated Regulation (EU) 2016/127 of 25

September 2015 supplementing Regulation (EU) No 609/2013 of the European Parliament and of the Council as regards the specific compositional and information requirements for infant formula and follow-on formula and as regards requirements on information relating to infant and young child feeding (Text with EEA relevance). In: Union E, ed. EU 2016/127. Official Journal of the European Union: European Union; 2016.

25. Vandentorren S, Bois C, Pirus C, et al. Rationales, design and recruitment for the Elfe longitudinal study. BMC Pediatr. 2009;9:58.

26. Koletzko S, Niggemann B, Arato A, et al. Diagnostic approach and management of cow's-milk protein allergy in infants and children: ESPGHAN GI Committee practical guidelines. J Pediatr Gastroenterol Nutr. 2012;55(2):221-229.

27. Bournez M, Ksiazek E, Wagner S, et al. Factors associated with the introduction of complementary feeding in the French ELFE cohort study. Matern Child Nutr.

2018;14(2):e12536. 
28. Juillard H. Weighting of Elfe survey data at time 0. In. pandora.vjf.inserm.fr/public/2015.

29. Hoppu U, Kalliomaki M, Laiho K, Isolauri E. Breast milk--immunomodulatory signals against allergic diseases. Allergy. 2001;56 Suppl 67:23-26.

30. von Berg A, Koletzko S, Filipiak-Pittroff B, et al. Certain hydrolyzed formulas reduce the incidence of atopic dermatitis but not that of asthma: three-year results of the German Infant Nutritional Intervention Study. J Allergy Clin Immunol. 2007;119(3):718-725.

31. Dupont D, Boutrou R, Menard O, et al. Heat Treatment of Milk During Powder Manufacture Increases Casein Resistance to Simulated Infant Digestion. Food Digestion. 2010;1(1-2):28-39.

32. Allergologie SPdP. Asthme de l'enfant de moins de 36 mois : diagnostic, prise en charge et traitement en dehors des épisodes aigus. France: Haute Autorité de Santé;2009. 33. Bui DS, Walters HE, Burgess JA, et al. Childhood Respiratory Risk Factor Profiles and Middle-Age Lung Function: A Prospective Cohort Study from the First to Sixth Decade. Annals of the American Thoracic Society. 2018;15(9):1057-1066.

34. Belgrave DCM, Granell R, Turner SW, et al. Lung function trajectories from preschool age to adulthood and their associations with early life factors: a retrospective analysis of three population-based birth cohort studies. The Lancet Respiratory medicine. 2018;6(7):526-534.

35. Kilpelainen M, Terho EO, Helenius H, Koskenvuo M. Validation of a new questionnaire on asthma, allergic rhinitis, and conjunctivitis in young adults. Allergy. 2001;56(5):377-384.

36. Westman M, Lupinek C, Bousquet J, et al. Early childhood IgE reactivity to pathogenesis-related class 10 proteins predicts allergic rhinitis in adolescence. J Allergy Clin Immunol. 2015;135(5):1199-1206.e1191-1111.

37. Mata Fernandez C, Fernandez-Benitez M, Perez Miranda M, Guillen Grima F. Validation of the Spanish version of the Phase III ISAAC questionnaire on asthma. Journal of investigational allergology \& clinical immunology. 2005;15(3):201-210. 38. Vandenplas Y, Alarcon P, Fleischer D, et al. Should Partial Hydrolysates Be Used as Starter Infant Formula? A Working Group Consensus. J Pediatr Gastroenterol Nutr. 2016;62(1):22-35.

39. Allen KJ, Lowe AJ, Dharmage SC. The role of hydrolysates for atopy prevention-con. Pediatr Allergy Immunol. 2013;24(8):724-726.

40. von Berg A. The role of hydrolysates for allergy prevention--pro. Pediatr Allergy Immunol. 2013;24(8):720-723.

41. von Berg A, Koletzko S, Grubl A, et al. The effect of hydrolyzed cow's milk formula for allergy prevention in the first year of life: the German Infant Nutritional Intervention Study, a randomized double-blind trial. J Allergy Clin Immunol. 2003;111(3):533-540. 
Table 1: Sample characteristics according to 2-month milk feeding, in the complete case sample $(\mathrm{N}=11,720)$

\begin{tabular}{lcccc} 
& nHF & pHF-non HA & pHF-HA & Breast milk only \\
\hline $\mathrm{N}$ & 7149 & 239 & 251 & 4081 \\
Maternal age at first delivery (y, mean (SD)) & $27.3(4.7)$ & $27.4(4.5)$ & $28.2(4.6)$ & $28.2(4.2)$ \\
Maternal master degree (\% (n)) & $16.1 \%(1150)$ & $16.7 \%(40)$ & $21.5 \%(54)$ & $28.4 \%(1161)$ \\
Family income per consumption unit (€, mean (SD)) & $1650(1585)$ & $1639(865)$ & $1860(864)$ & $1752(1272)$ \\
Both parents born in France (\% (n)) & $84.3 \%(6027)$ & $84.1 \%(201)$ & $89.2 \%(224)$ & $80.3 \%(3278)$ \\
Maternal smoking during pregnancy (\% (n)) & & & \\
$\quad$ Never smoker & $84.3 \%(6027)$ & $84.1 \%(201)$ & $89.2 \%(224)$ & $80.3 \%(3278)$ \\
$\quad$ Smoker only before pregnancy & $7.6 \%(541)$ & $7.9 \%(19)$ & $5.2 \%(13)$ & $7.7 \%(316)$ \\
$\quad$ Smoker only in early pregnancy & $4.5 \%(320)$ & $4.2 \%(10)$ & $3.6 \%(9)$ & $7 \%(287)$ \\
$\quad$ Smoker throughout pregnancy & $3.7 \%(261)$ & $3.8 \%(9)$ & $2 \%(5)$ & $4.9 \%(200)$ \\
No passive smoking (\% (n)) & $72.6 \%(5192)$ & $73.6 \%(176)$ & $76.1 \%(191)$ & $82.7 \%(3377)$ \\
Residence in rural area (<2000 inhab, \% (n)) & $23.9 \%(1707)$ & $21.3 \%(51)$ & $22.3 \%(56)$ & $21.2 \%(866)$ \\
C-section delivery (\% (n)) & $19.1 \%(1364)$ & $24.3 \%(58)$ & $15.5 \%(39)$ & $14.3 \%(584)$ \\
No parental history of allergy (\% (n)) & $51.2 \%(3661)$ & $43.1 \%(103)$ & $28.7 \%(72)$ & $46.8 \%(1910)$ \\
Sibling history of allergy (\% (n)) & & & \\
$\quad$ No sibling & $46.9 \%(3354)$ & $46.9 \%(112)$ & $48.6 \%(122)$ & $39.5 \%(1611)$ \\
$\quad$ No history of allergy in siblings & $40.3 \%(2884)$ & $36 \%(86)$ & $31.5 \%(79)$ & $45.4 \%(1851)$ \\
$\quad$ History of allergy in siblings & $12.7 \%(911)$ & $17.2 \%(41)$ & $19.9 \%(50)$ & $15.2 \%(619)$ \\
Boys (\% (n)) & $50.5 \%(3612)$ & $52.7 \%(126)$ & $61 \%(153)$ & $50.8 \%(2074)$ \\
Gestational age (weeks, mean (SD)) & $39.2(1.5)$ & $39.1(1.6)$ & $39.3(1.3)$ & $39.4(1.3)$ \\
Breastfed at 2 months (\% (n)) & $24.2 \%(1729)$ & $20.1 \%(48)$ & $40.2 \%(101)$ & $100 \%(4081)$ \\
No change in formula milk from birth $(\%(\mathrm{n}))$ & $53.2 \%(3805)$ & $22.2 \%(53)$ & $74.1 \%(186)$ & $100 \%(4081)$ \\
Treatment for GERD reported at 2 months (\% (n)) & $24.3 \%(1735)$ & $61.1 \%(146)$ & $16.3 \%(41)$ & $10.5 \%(430)$ \\
Age at complementary feeding introduction (months, & & & \\
mean (SD)) & $5.2(1.1)$ & $5.0(1.2)$ & $5.3(1.1)$ & $5.7(1.0)$ \\
\hline
\end{tabular}

nHF: non-hydrolysed formula; pHF-non-HA: partially hydrolysed formula without hypoallergenic label; pHF-HA: partially hydrolysed formula with hypoallergenic label. Infants receiving both breast and formula milk were classified according to the infant formula consumed. 
Table 2: Prevalence of allergy-related symptoms up to 2 years

\begin{tabular}{lccc} 
& 2 months & 1 year & 2 years \\
\hline $\mathbf{N}$ & 15,460 & 13,384 & 12,363 \\
Parental reported of medical diagnosis of eczema & $16.1 \%(2495)$ & $17.8 \%(2387)$ & $18.5 \%(2291)$ \\
Parental report of respiratory symptoms & & & $18.7 \%(2317)$ \\
$\quad$ Wheezing & $6.7 \%(1028)$ & $27.3 \%(3650)$ & $6.1 \%(757)$ \\
$\quad$ Asthma attack in the previous 12 months & $/$ & $/$ & $/$ \\
Parental report of food allergy & & $/$ & $4.1 \%(511)$ \\
$\quad$ Medical diagnosis of CMPA & $1.5 \%(227)$ & & $97.1 \%(7046)$ \\
$\quad$ Medical advice of food eviction due to allergy & & $/$ & $1.7 \%(125)$ \\
Physician report of food allergy (sub-sample, n=7258) & $/$ & $/$ & $1.2 \%(87)$
\end{tabular}




\section{Figure legend}

Figure 1: Sample selection

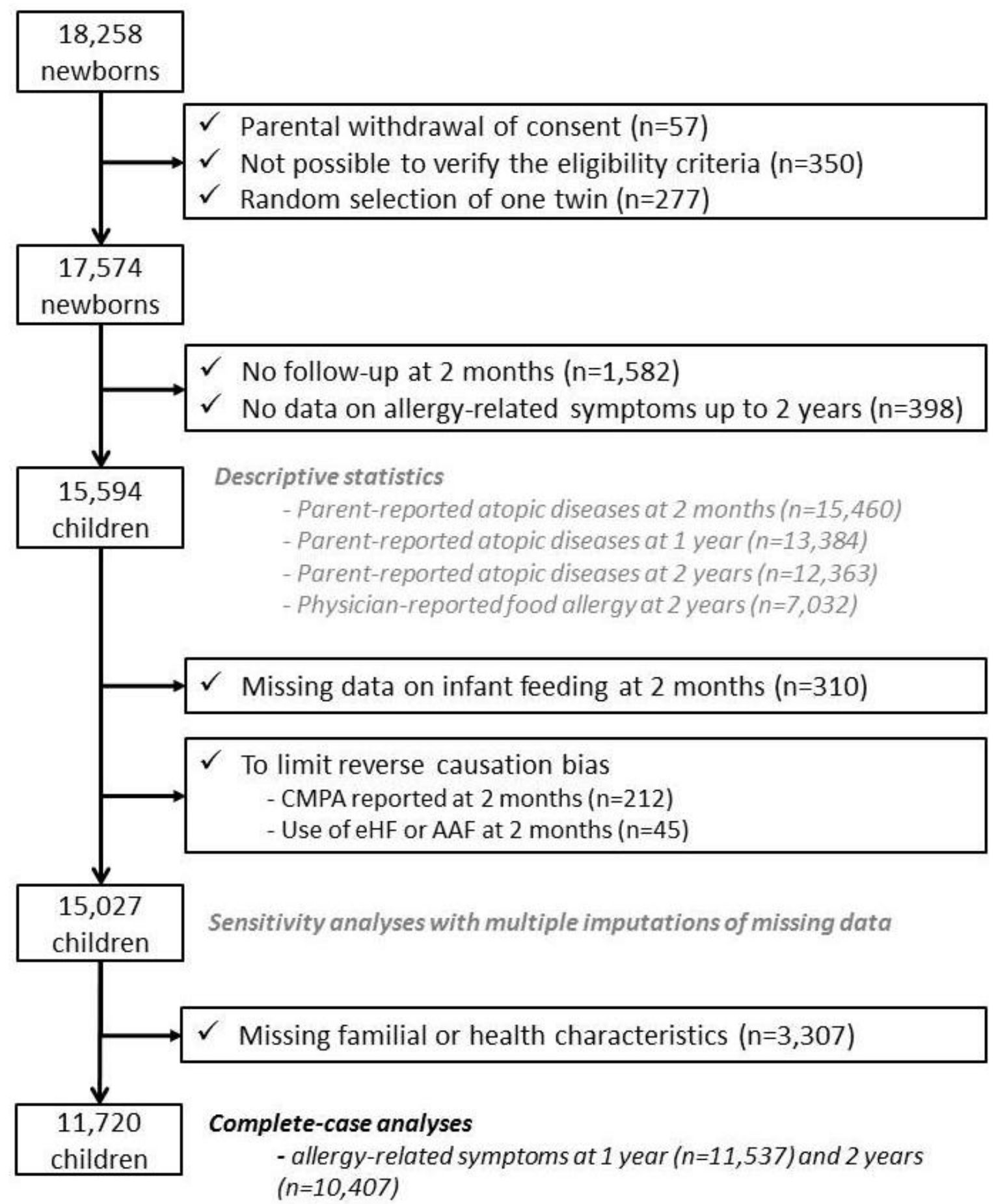

Footnote: Description of the successive steps by which infants were excluded to reach the complete case analysis sample size. CMPA: Cow's milk protein allergy; eHF: extensively hydrolysed formula; AAF: amino acids formula 
Figure 2: Adjusted associations between 2-month milk feeding and the incidence of eczema at 1 and 2 years and of food allergies at 2 years with non-hydrolysed formula as reference.

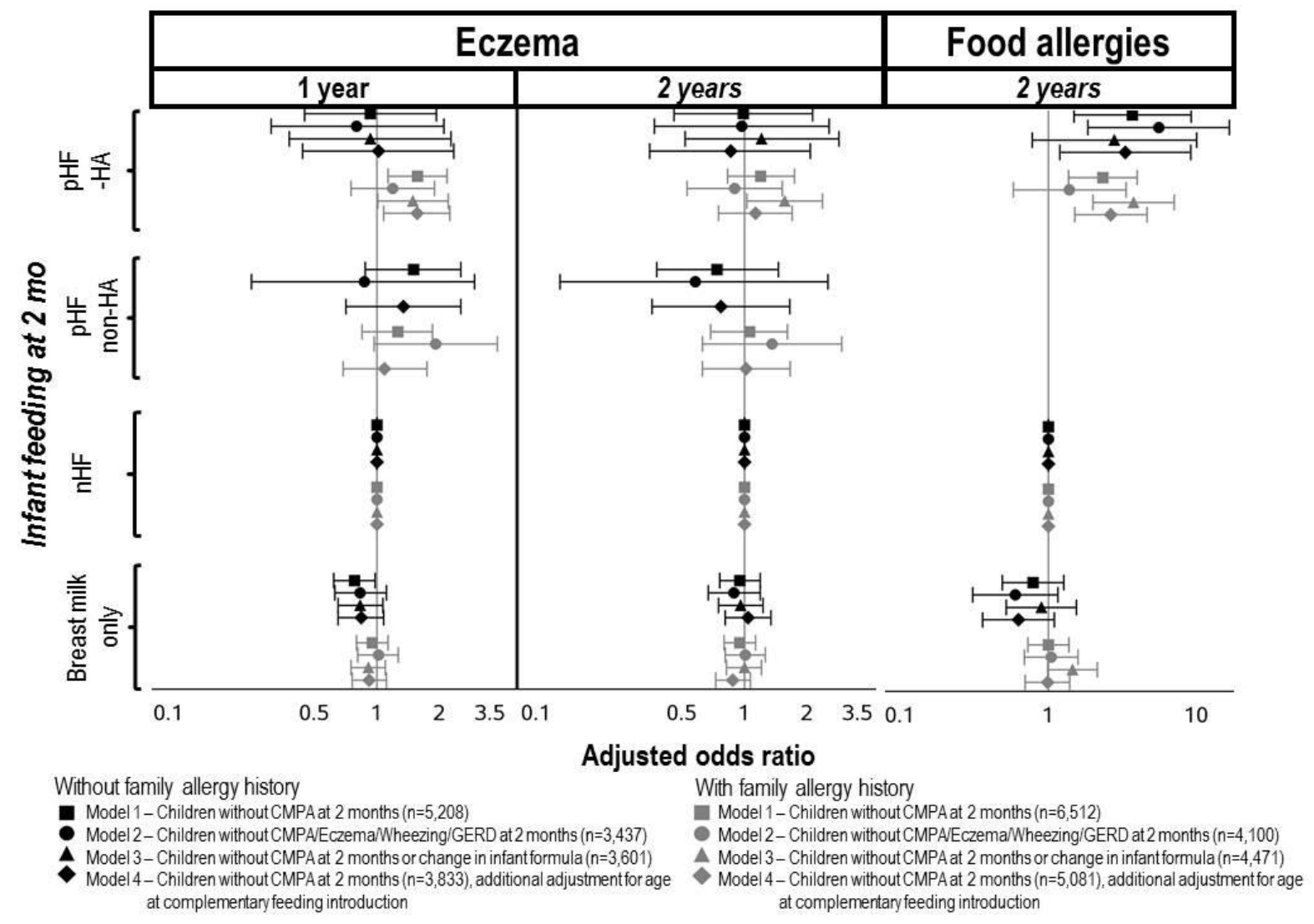

Legend: nHF: non-hydrolysed formula; pHF-non-HA: partially hydrolysed formula without hypoallergenic label; pHF-HA: partially hydrolysed formula with hypoallergenic label. Infants receiving both breast and formula milk were classified according to the infant formula consumed. Values are adjusted odds-ratio $(95 \% \mathrm{CI})$ from multinomial logistic regressions, with $\mathrm{nHF}$ as the reference group, adjusted for parental allergy history, sibling allergy history, age at first attendance at collective care arrangement, first physician seen after discharge, maternal education level and age at first child, parental place of birth, family monthly income per consumption unit, size of town of residence, maternal smoking status, passive smoking, gestational age at birth, sex, mode of delivery, any breastfeeding duration, number of people per room living in the household, recruitment wave, maternity unit size and level, and mother's region of residence. Among infants with familial history of allergy, analyses were also adjusted for the origin (parents or siblings) of this familial history of allergy. Infant receiving pHF-non-HA were excluded from analyses on food allergy and from Model 3, due to the limited sample size. 
Figure 3: Adjusted associations between 2-month milk feeding and the incidence of wheezing at 1 and 2 years and of asthma at 2 years, with non-hydrolysed formula as reference.

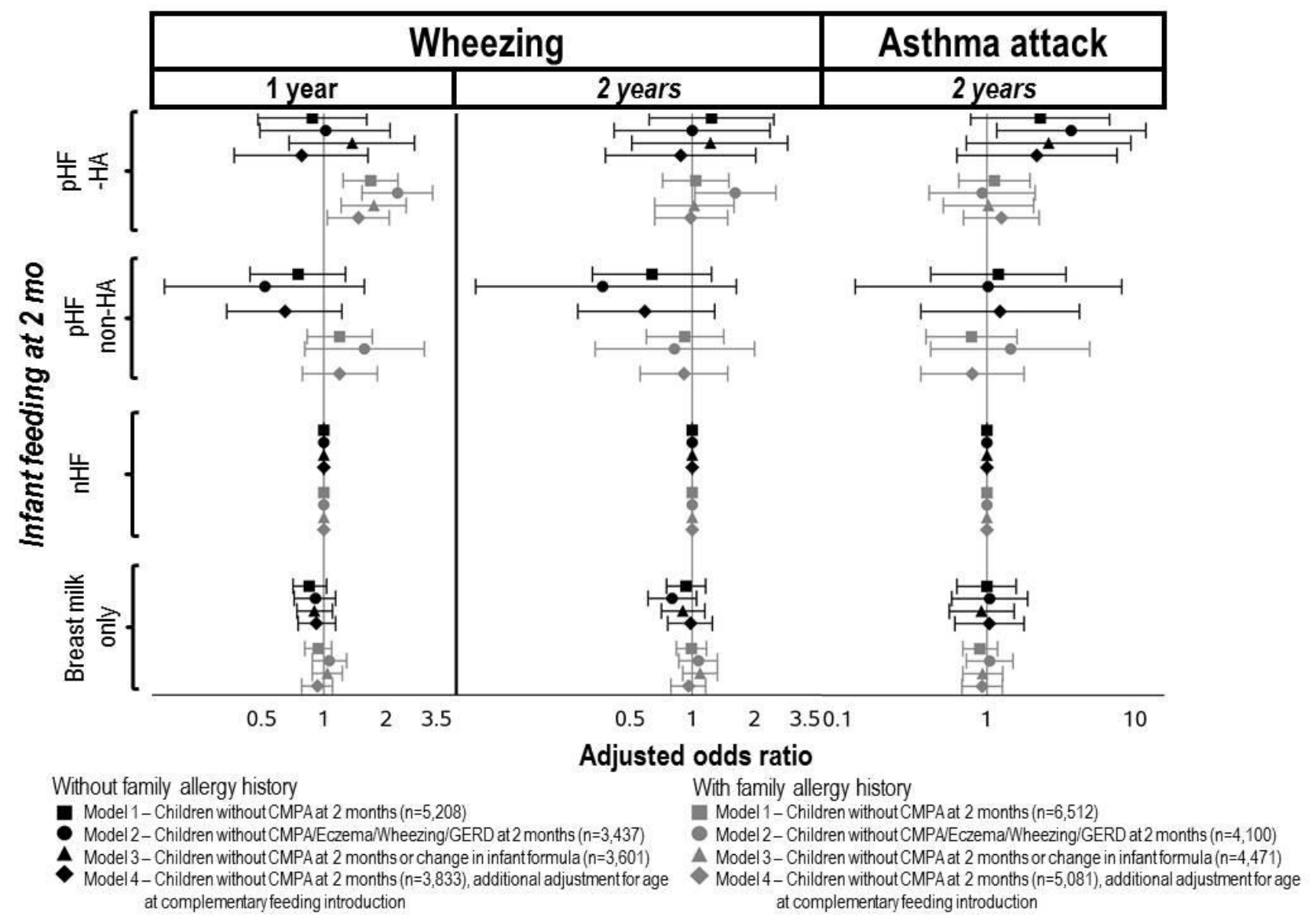


Legend: nHF: non-hydrolysed formula; pHF-non-HA: partially hydrolysed formula without hypoallergenic label; pHF-HA: partially hydrolysed formula with hypoallergenic label. Infants receiving both breast and formula milk were classified according to the infant formula consumed. Values are adjusted odds-ratio (95\%CI) from multinomial logistic regressions, with $\mathrm{nHF}$ as the reference group, adjusted for parental allergy history, sibling allergy history, age at first attendance at collective care arrangement, first physician seen after discharge, maternal education level and age at first child, parental place of birth, family monthly income per consumption unit, size of town of residence, maternal smoking status, passive smoking, gestational age at birth, sex, mode of delivery, any breastfeeding duration, number of people per room living in the household, recruitment wave, maternity unit size and level, and mother's region of residence. Among infants with familial history of allergy, analyses were also adjusted for the origin (parents or siblings) of this familial history of allergy. Infant receiving pHF-non-HA were excluded from analyses on food allergy and from Model 3, due to the limited sample size. 


\section{APPENDICES}

Supplementary method 1: Data collection process regarding adjustment variables

Familial allergy history was collected at the 2-month interview. Parents and siblings were considered to have a history of allergy when they were reported to ever have eczema, asthma, or hay fever. Family history of FA was unfortunately not collected. Parental history of allergy was treated as a four-category variable: $1 /$ no parental history of allergy, 2 / history of maternal allergy only, 3/ history of paternal allergy only and 4/ both parents with history of allergy. Sibling history of allergy was considered as a three-category variable: 1/ no sibling, 2/ sibling with no history of allergy, $3 /$ at least one sibling with a history of allergy. Total breastfeeding duration was computed prior to the present analyses, from all interviews and questionnaires from birth to 2 years ${ }^{23}$. Maternal smoking status during pregnancy (never smoked, only before pregnancy, only in early pregnancy, throughout pregnancy) and maternal exposure to second-hand smoke (none, outside home, at home, both outside and at home) were collected at delivery. Mode of delivery, child's sex, twin birth, and gestational age were collected from the medical record. Total breastfeeding duration, i.e. period during which the infant received breastmilk regardless of exclusivity, was computed from all questionnaires from birth to 2 years of age. Age at first attendance to a collective childcare arrangement ( 3 children or more on site) was collected in the 1-y interview and considered as a four-category variable ( $\leq 4$ months, $>4$ to 8 months, $>8$ to 12 months, never attended at 12 months).

Supplementary method 2: Details regarding sensitivity analyses

To address the indication bias issue, a first sensitivity analysis (Model 2) was conducted after exclusion of infants with symptoms related to allergic diseases (wheezing, eczema or treatment for gastroesophageal reflux disease (GERD)) reported at the 2-month follow-up. A second sensitivity analysis (Model 3) was conducted only among infants without any change in infant formula from infant formula introduction to the 2-month follow-up. 
To account for other aspects of infant feeding, a third sensitivity analysis (Model 4) was conducted in the sample as the main analysis but further adjusted for age at complementary food introduction. Results from these 3 additional models are displayed on figure 2 and figure 3.

At the 2-year interview, a physician questionnaire was available. Physician could report whether FA was diagnosed and, when relevant, whether IgE or skin test was used for this diagnosis. As the physician-questionnaire was not completed for all children, it was not considered in the main analyses but only in sensitivity analyses. Physician-report of FA was considered as a 3-category variable (no allergy, without use of IgE or skin test to confirm FA, with use of IgE or skin test to confirm FA). The sample size did not allow stratifying the analysis on family allergy history but we adjusted for both parental and sibling history of allergy.

As the only pHF with documented efficacy regarding the prevention of allergy-related symptoms was whey-based ${ }^{41}$ and consumed in the first 4 months of life, two additional sensitivity analyses were conducted The first one was restricted to whey-based pHF, regardless of HA label, at 2 months. The second one assessed the associations between infant feeding at 4 months and allergyrelated symptoms, but data collection did not allow excluding cases diagnosed between 2 and 4 months but only those reported at 2 months.

To deal with missing measurements in sociodemographic variables, we conducted multiple imputations using the SAS software. We assumed that data were missing at random and generated five independent datasets with the fully conditional specification method (MI procedure, FCS statement, NIMPUTE option), and then calculated pooled effect estimates (SAS MIANALYSE procedure). Further details are available in supplementary table 3. 
Supplementary Table 1: Comparison of families included in the 'complete case' analysis with other families in the ELFE study (Chi2 test comparison, Student t-test comparison)

\begin{tabular}{|c|c|c|}
\hline & Included & Non-selected \\
\hline \multicolumn{3}{|l|}{ Feeding at birth } \\
\hline Breast milk only & $65.7 \%(7773)$ & $53.3 \%(3388)$ \\
\hline Formula milk only & $25.1 \%(2971)$ & $32.7 \%(2079)$ \\
\hline Breast and formula milk & $8.9 \%(1053)$ & $13.5 \%(860)$ \\
\hline Other milk & $0.2 \%(24)$ & $0.4 \%(23)$ \\
\hline Breast and other milk & $0.0 \%(4)$ & $0.0 \%(2)$ \\
\hline \multicolumn{3}{|l|}{ Sex } \\
\hline Girl & $49.1 \%(5803)$ & $47.8 \%(2967)$ \\
\hline Boy & $50.9 \%(6026)$ & $52.2 \%(3241)$ \\
\hline \multicolumn{3}{|l|}{ Gestational age } \\
\hline$<37 \mathrm{WA}$ & $4.4 \%(523)$ & $7.6 \%(463)$ \\
\hline [37 - 41] WA & $81.4 \%(9629)$ & $80.1 \%(4895)$ \\
\hline$>41 \mathrm{WA}$ & $14.2 \%(1677)$ & $12.3 \%(751)$ \\
\hline \multicolumn{3}{|l|}{ Mode of delivery } \\
\hline Vaginal & $82.5 \%(9757)$ & $78.8 \%(4651)$ \\
\hline C-section & $17.5 \%(2072)$ & $21.2 \%(1255)$ \\
\hline \multicolumn{3}{|l|}{ Maternal age } \\
\hline$<25$ years & $10.3 \%(1216)$ & $18.4 \%(1177)$ \\
\hline [25 - 29] years & $32.4 \%(3835)$ & $31.7 \%(2026)$ \\
\hline$[30-34]$ years & $36.9 \%(4367)$ & $30.1 \%(1922)$ \\
\hline$>34$ years & $20.4 \%(2411)$ & $19.9 \%(1270)$ \\
\hline \multicolumn{3}{|l|}{ Education level } \\
\hline Below secondary school & $5.8 \%(682)$ & $13.7 \%(628)$ \\
\hline Secondary school & $13.1 \%(1547)$ & $19.1 \%(876)$ \\
\hline High school & $18.3 \%(2160)$ & $19.8 \%(910)$ \\
\hline 2-y university degree & $23.5 \%(2781)$ & $17.7 \%(812)$ \\
\hline 3-y university degree & $18.9 \%(2232)$ & $12.9 \%(594)$ \\
\hline 5-y university degree or more & $20.5 \%(2427)$ & $16.8 \%(771)$ \\
\hline \multicolumn{3}{|l|}{ Parental country of birth } \\
\hline Both parents born in France & $83.0 \%(9822)$ & $68.5 \%(3225)$ \\
\hline Mother born in France only & $7.6 \%(897)$ & $12.4 \%(585)$ \\
\hline Father born in France only & $5.3 \%(631)$ & $8.4 \%(397)$ \\
\hline No parent born in France & $4.0 \%(479)$ & $10.7 \%(502)$ \\
\hline \multicolumn{3}{|l|}{ Maternal smoking } \\
\hline Never smoker & $56.6 \%(6694)$ & $58.3 \%(3582)$ \\
\hline Smoker only before pregnancy & $24.9 \%(2940)$ & $18.5 \%(1136)$ \\
\hline Smoker only in early pregnancy & $3.8 \%(452)$ & $4.3 \%(265)$ \\
\hline Smoker throughout pregnancy & $14.7 \%(1743)$ & $18.9 \%(1164)$ \\
\hline
\end{tabular}

All p-value $<0.0001$, except for sex (p-value $=0.11)$ 
Supplementary table 2: Bivariate analyses of 2-month milk feeding and allergy or respiratory symptoms at 1 and 2 years (Chi2 test comparison)

Eczema

\begin{tabular}{ccc}
\cline { 2 - 3 } & \multicolumn{2}{c}{1 year } \\
\cline { 2 - 3 } & No & Yes \\
\hline Infants without familial history of allergy & & \\
2-mo infant feeding & $33.8 \%(1477)$ & $29.3 \%(218)$ \\
Breast milk only & $63.5 \%(2774)$ & $66.9 \%(497)$ \\
nHF & $1.6 \%(69)$ & $2.6 \%(19)$ \\
pHF-non-HA & $1.2 \%(51)$ & $1.2 \%(9)$
\end{tabular}

Infants with familial history of allergy

2-mo infant feeding

Breast milk only

nHF

pHF-non-HA

pHF-HA

0.04

\begin{tabular}{lrrr}
\multicolumn{4}{c}{2 years } \\
$\mathrm{p}$ & No & Yes & $\mathrm{p}$ \\
\hline
\end{tabular}

\begin{tabular}{crc}
\multicolumn{3}{c}{ Food allergy } \\
\hline \multicolumn{3}{c}{2 years } \\
\hline No & Yes & $\mathrm{p}$ \\
\hline
\end{tabular}

$$
\begin{array}{cc}
34.4 \%(1325) & 35.2 \%(240) \\
62.6 \%(2412) & 62.1 \%(423) \\
1.9 \%(73) & 1.5 \%(10) \\
1.1 \%(43) & 1.2 \%(8)
\end{array}
$$

0.86

$34.3 \%(1520) \quad 31.9 \%(45)$

$62.8 \%(2786) \quad 62.4 \%(88)$ $1.9 \%(83) \quad 1.4 \%(2)$

$1 \%(45) \quad 4.3 \%(6)$
.0005

$37.7 \%(1743) \quad 38.1 \%(475)$

$57.3 \%$ (2649) $56.1 \%$ (700)

$2.3 \%(105) \quad 2.4 \%(30)$

$2.8 \%(129) \quad 3.4 \%(42)$
.004

$37.1 \%(1886) \quad 34 \%(455)$

$58.2 \%(2960) \quad 58.8 \%(788)$

$2.1 \%(107) \quad 2.8 \%(38)$

$2.6 \%(130) \quad 4.4 \%(59)$
0.69

$37.3 \%$ (2098) $40.4 \%$ (120)

\begin{tabular}{|c|c|c|c|c|c|c|c|c|}
\hline & \multicolumn{5}{|c|}{ Wheezing } & \multicolumn{3}{|c|}{ Asthma } \\
\hline & \multicolumn{2}{|c|}{1 year } & \multicolumn{3}{|c|}{2 years } & \multicolumn{3}{|c|}{2 years } \\
\hline & No & Yes & No & Yes & $\mathrm{p}$ & No & Yes & $\mathrm{p}$ \\
\hline \multicolumn{9}{|c|}{ Infants without familial history of allergy } \\
\hline 2-mo infant feeding & & & 0.63 & & 0.59 & & & .004 \\
\hline Breast milk only & $33.5 \%(1292)$ & $31.9 \%(403)$ & $34.7 \%(1313)$ & $33.6 \%(252)$ & & $34.6 \%(1520)$ & $32.1 \%(45)$ & \\
\hline $\mathrm{nHF}$ & $63.5 \%(2446)$ & $65.4 \%(825)$ & $62.3 \%(2359)$ & $63.5 \%(476)$ & & $62.5 \%(2748)$ & $62.1 \%(87)$ & \\
\hline pHF-non-HA & $1.8 \%(69)$ & $1.5 \%(19)$ & $1.9 \%(72)$ & $1.5 \%(11)$ & & $1.8 \%(81)$ & $1.4 \%(2)$ & \\
\hline pHF-HA & $1.2 \%(45)$ & $1.2 \%(15)$ & $1.1 \%(40)$ & $1.5 \%(11)$ & & $1 \%(45)$ & $4.3 \%(6)$ & \\
\hline \multicolumn{9}{|c|}{ Infants with familial history of allergy } \\
\hline 2-mo infant feeding & & & .0001 & & 0.84 & & & .003 \\
\hline Breast milk only & $37.4 \%(1668)$ & $34.2 \%(673)$ & $37.7 \%(1725)$ & $38 \%(493)$ & & $37.6 \%(2098)$ & $40.7 \%(120)$ & \\
\hline $\mathrm{nHF}$ & $58.1 \%(2589)$ & $59 \%(1159)$ & $57.2 \%(2616)$ & $56.6 \%(733)$ & & $57.3 \%(3197)$ & $51.5 \%(152)$ & \\
\hline pHF-non-HA & $2.1 \%(95)$ & $2.5 \%(50)$ & $2.3 \%(107)$ & $2.2 \%(28)$ & & $2.3 \%(130)$ & $1.7 \%(5)$ & \\
\hline pHF-HA & $2.4 \%(106)$ & $4.2 \%(83)$ & $2.8 \%(129)$ & $3.2 \%(42)$ & & $2.7 \%(153)$ & $6.1 \%(18)$ & \\
\hline
\end{tabular}

$57.6 \%(3241) \quad 51.9 \%(154)$

$2.4 \%(134) \quad 1.7 \%(5)$

$2.8 \%(156) \quad 6.1 \%(18)$ 
Supplementary Table 3: Details regarding multiple imputations

Variable

Type of variable

Model used to Missin

predict missing $\mathrm{g}$

data

values

Maternal employment

Binary

No missing data

$0 \%$

Wave of recruitment

Categorical (4

categories)

Parental country of birth

Categorical (4

categories)

Mother's region of residence

Maternal age at delivery

Gestational age

Parity

Predominant breast feeding duration

Maternity unit level

Categorical $(9$

categories)

No missing data $\quad 0 \%$

Continuous

Continuous

Continuous

Continuous

Ordinal (3 categories)

Ordinal (3 categories)

Sibling history of allergy

Infant feeding at 2 months

Maternity unit size

City size

Maternal highest diploma

Change in infant formula reported at 2

months

Ordinal (4 categories)

Ordinal (5 categories)

Ordinal (4 categories)

No missing data

$0 \%$

No missing data

$0 \%$

No missing data

$0 \%$

No missing data

$0 \%$

No missing data

$0 \%$

No missing data $\quad 0 \%$

No missing data $\quad 0 \%$

No missing data $\quad 0 \%$

No missing data $\quad 0 \%$

No missing data $\quad 0 \%$

Ordinal (6 categories)

Logistic regression

$0 \%$

Logistic regression

$0 \%$

Diagnostic of CMPA at 2 months

Wheezing at 2 months

Binary

Logistic regression

$0 \%$

Binary

Logistic regression

$0 \%$

Binary

Logistic regression

$0 \%$

Binary

Logistic regression

$0 \%$

Sex

Binary

Logistic regression

$0 \%$

Binary

Logistic regression

$0 \%$

Continuous

Linear regression

$0 \%$

Ordinal (4 categories)

Logistic regression

$0 \%$

Parental history of allergy

Ordinal (5 categories)

Logistic regression

$0 \%$

Continuous

Categorical (5

Linear regression

$1 \%$

First physician seen after discharge

Maternal smoking during pregnancy

categories)

Categorical (4

Multinomial

regression

$1 \%$

categories)

Multinomial

regression

Continuous

Linear regression

$1 \%$

Continuous

Linear regression

$1 \%$

Binary

Logistic regression

$2 \%$

Family monthly income per consumption

unit

Second-hand smoking

Wheezing at 1 year

Eczema at 1 year

Age at first attendance to collective care

Wheezing at 2 years

Asthma at 2 years

Continuous

Linear regression

Ordinal (4 categories)

Binary

Logistic regression

Binary

Logistic regression

Ordinal (4 categories)

Logistic regression

Binary

Logistic regression

Binary

Logistic regression

Logistic regression

Binary

Logistic regression

Logistic regression 
Physician-reported respiratory allergy at 2 years 
Supplementary table 4: Sensitivity analyses (whey-based pHF, infant feeding at 4 months, multiple imputations) on eczema and food allergy.

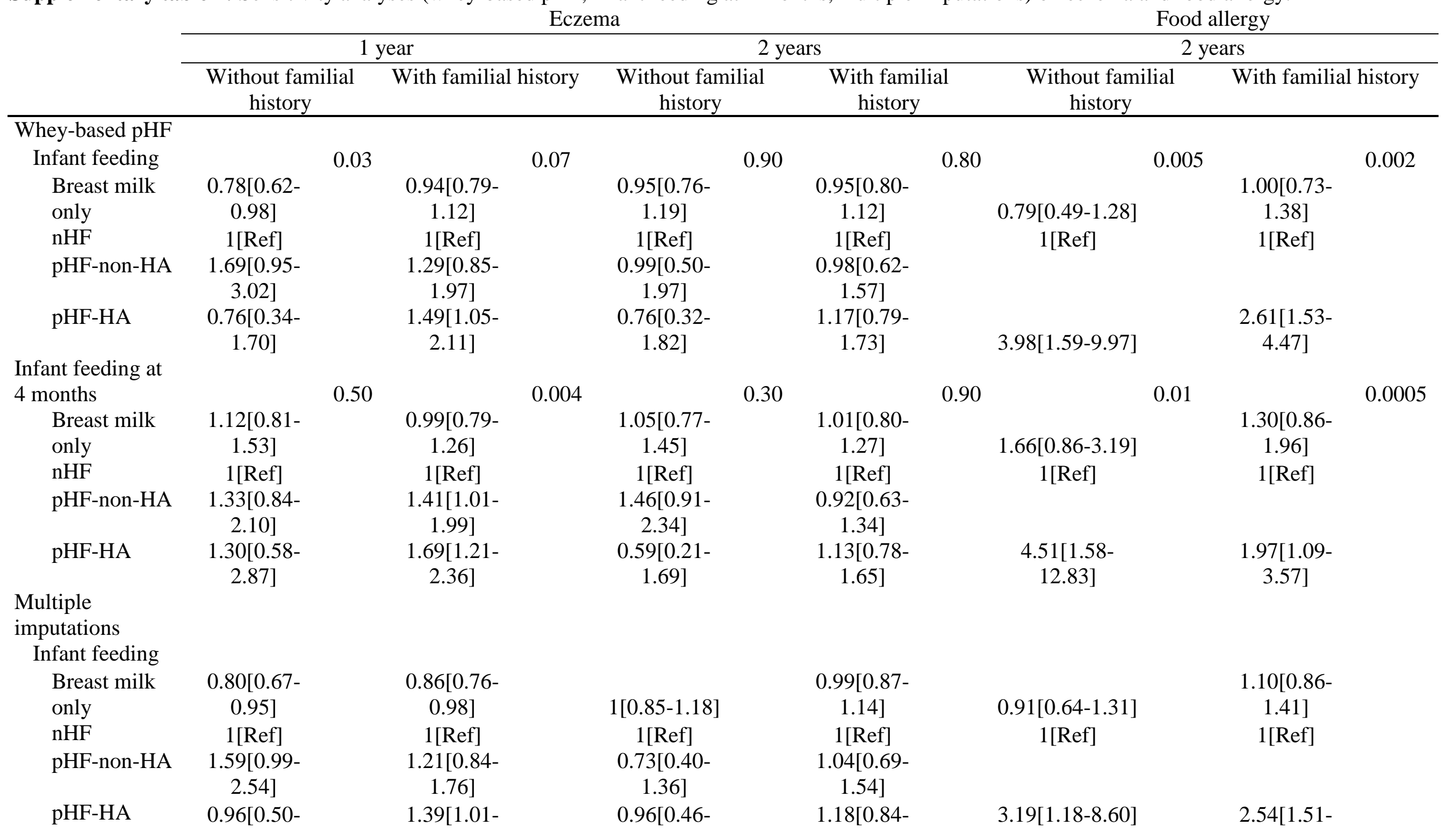


$1.86]$

1.90]

1.99]

$1.64]$

4.27]

Values are adjusted odds-ratio (95\%CI) from multinomial logistic regressions, with nHF as the reference group, adjusted for parental allergy history, sibling allergy history, age at first attendance at collective care arrangement, first physician seen after discharge, maternal education level and age at first child, parental place of birth, family monthly income per consumption unit, size of town of residence, maternal smoking status, passive smoking, gestational age at birth, sex, mode of delivery, any breastfeeding duration, number of people per room living in the household, recruitment wave, maternity unit size and level, and mother's region of residence. Among infants with familial history of allergy, analyses were also adjusted for the origin (parents or siblings) of this familial history of allergy. Infant receiving pHF-non-HA were excluded from analyses on food allergy and from Model 3, due to the limited sample size. 
Supplementary table 5: Sensitivity analyses (whey-based pHF, infant feeding at 4 months, multiple imputations) on respiratory symptoms.

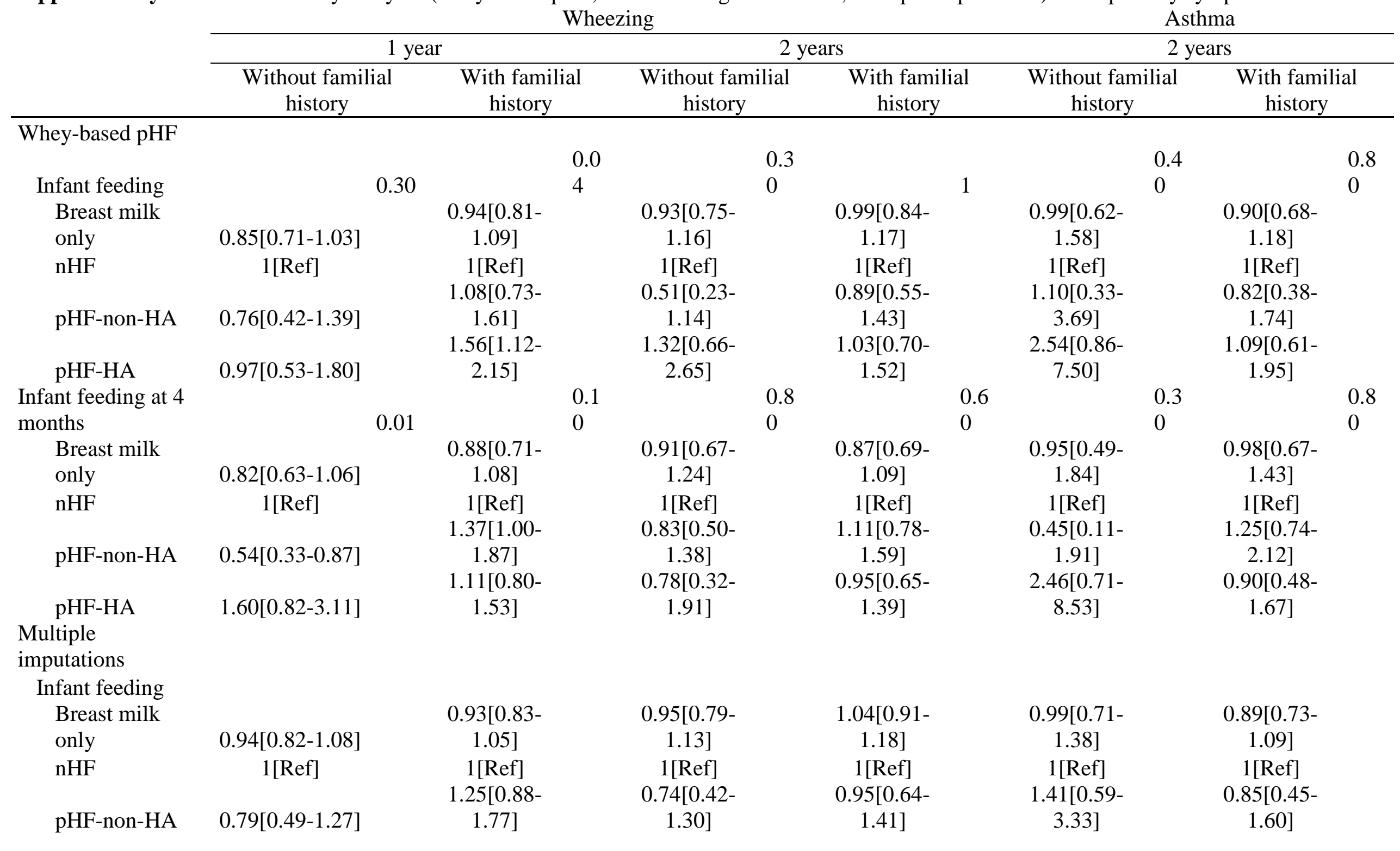



pHF-HA
$1.04[0.61-1.78]$
$1.62[1.21-$
$1.33[0.69-$
$1.20[0.86-$
$2.65[1.10-$
6.42]
1.23[0.72-
1.67]
2.11]

Values are adjusted odds-ratio (95\%CI) from multinomial logistic regressions, with nHF as the reference group, adjusted for parental allergy history, sibling allergy history, age at first attendance at collective care arrangement, first physician seen after discharge, maternal education level and age at first child, parental place of birth, family monthly income per consumption unit, size of town of residence, maternal smoking status, passive smoking, gestational age at birth, sex, mode of delivery, any breastfeeding duration, number of people per room living in the household, recruitment wave, maternity unit size and level, and mother's region of residence. Among infants with familial history of allergy, analyses were also adjusted for the origin (parents or siblings) of this familial history of allergy. Infant receiving pHF-non-HA were excluded from analyses on food allergy and from Model 3, due to the limited sample size 
Supplementary material 1: Items used to define parental-report of eczema, respiratory symptoms and food allergies in the ELFE study

- 2-month interview

○ Has a doctor diagnosed an allergy to cow's milk proteins? Yes/No

- 1-year interview

- Has your child been diagnosed with eczema? Yes/No

○ Has your child had wheezing in the chest in the past 12 months? Yes/No

- 2-year interview

- Has your child had any eczema flare-ups in the last 12 months? Yes/No

O On the advice of a doctor, have you stopped giving your child certain foods owing to a food allergy?

- Has your child had at least one episode of wheezing in the chest in the last 12 months? Yes/No

- Has your child had any asthma attacks in the last 12 months? Yes/No 